\title{
Triagem e adequação do fluxo de pacientes no departamento de emergência de um hospital terciário durante a pandemia de COVID-19: relato de experiência
}

\author{
Screening and adaptation of patient flow in the emergency \\ department of a tertiary hospital during the COVID-19 pandemic: \\ experience report
}

\author{
Breno Douglas \\ Dantas Oliveiral,* (iD \\ Samer Heluany Khoury" iD \\ Vanessa Gomes Martins" (iD) \\ Frederico Carlos de \\ Sousa Arnaud'
}

Ane Caroline Gaspardi"I

Dieison Roberto

Vieira Rabêlo" iD

\begin{abstract}
RESUMO
Introdução: A pandemia de COVID-19 representa um dos maiores desafios sanitários da atualidade e apresenta-se clinicamente de forma variada quanto aos sintomas e gravidade. A partir de sua forma de contaminação por aerossóis e fômites, o avanço da doença gera crescente busca por atendimento nos serviços de emergência, trazendo aos gestores o desafio de garantir recursos humanos e materiais para essa demanda, assim como definir fluxos seguros para evitar contaminações nosocomiais, protegendo pacientes e trabalhadores da saúde. Objetivo: Relatar uma experiência de adequação do fluxo no atendimento de pacientes suspeitos de COVID-19, com auxílio de sistema informatizado adaptado ao procedimento de triagem. Método: Trata-se de um estudo descritivo realizado em um hospital terciário público do estado do Ceará, a partir da observação e discussão das estratégias de enfrentamento a pandemia de COVID-19 pela equipe de gestores do departamento de emergência. Resultados: A partir do acolhimento e triagem dos pacientes, a adequação dos serviços de saúde em fluxos diferenciados para o paciente não suspeito e suspeito de COVID-19 representa uma ferramenta importante na garantia de segurança e resolutividade no enfrentamento da pandemia de COVID-19. Conclusões: A criação de uma triagem extra-hospitalar utilizando uma ferramenta informatizada pode ser evidenciada como uma estratégia na otimização dos recursos hospitalares, evitando a admissão de pacientes que poderiam ter suas demandas atendidas em serviços de menor complexidade ou mesmo em seus domicílios, proporcionando maior agilidade no seguimento dos fluxos.
\end{abstract}

PALAVRAS-CHAVE: Emergência; Triagem; COVID-19
Universidade de Fortaleza, Fortaleza, CE, Brasil

" Hospital de Messejana, Fortaleza, CE, Brasil

III Escola Cearense de Emergências Médicas, Fortaleza, CE, Brasil

\footnotetext{
* E-mail: brenodouglas@hotmail.com
}

Recebido: 25 maio 2020 Aprovado: 03 jun 2020

\begin{abstract}
Introduction: The COVID-19 pandemic represents one of the greatest health challenges today and has a variety of symptoms and severity. From its form of contamination by aerosols and fomites, the progress of the disease represents an increased demand for assistance in emergency services, challenging managers to guarantee human and material resources for this demand, as well as defining safe flows to avoid nosocomial contamination, protecting patients and health workers. Objective: To report a flow adjustment experience of patients suspected of COVID-19, aided by a computerized system adapted to the screening procedure. Method: This is a descriptive study carried out in a public hospital in the state of Ceará - Brazil, based on the observation and discussion of strategies to deal with the COVID-19 pandemic by the management team in the emergency department. Results: From the reception and screening of patients, an adaptation of health services in differentiated flows to the non-suspect and suspected patient of COVID-19 represents an important strategy in guaranteeing security and resolvability in coping with the pandemic of COVID-19. Conclusions: The creation of an extrahospital screening, using a computerized tool, can be evidenced as a strategy to optimize hospital resources, avoiding the admission of patients with demands belonging to less complex health services or even at home, giving greater agility in monitoring hospital flows.
\end{abstract}

KEYWORDS: Emergency; Screening; COVID-19 


\section{INTRODUÇÃO}

A COVID-19 é uma pandemia viral descrita em mais de 120 países, causada pelo Coronavírus SARS-CoV-2 e que se apresenta com manifestações clínicas variadas, de casos leves a severos, incluindo a morte ${ }^{1}$. Desde o seu início na China em dezembro de 2019, a pandemia de COVID-19 vem representando um dos maiores desafios sanitários deste século².

No Brasil, o Ministério da Saúde (MS) reporta em seus meios oficiais de comunicação 584.016 casos confirmados até 03 de junho de 2020, com relatos em todos os estados brasileiros e com taxa de letalidade de $5,6 \%$. O estado do Ceará registra 56.056 casos confirmados com 3.605 óbitos pela doença3 ${ }^{3}$. Em uma população na qual aproximadamente $76 \%$ dos habitantes utilizam apenas o Sistema Único de Saúde, gerir uma pandemia em contextos de aglomerações urbanas e fragilidade da rede em regiões rurais demanda aos gestores a necessidade de criar novos fluxos e regimes estratégicos de triagem na emergência, com intuito de otimizar os acessos e dimensionar recursos ${ }^{4}$.

O objetivo deste estudo foi descrever as estratégias de mudança de fluxo dos pacientes com suspeita de COVID-19, desde a triagem até setores de internação, em um hospital terciário, como ferramenta de proteção de usuários e trabalhadores, diminuindo o contato e, portanto, o risco de contaminação nosocomial. Além disso, a partir de uma triagem extra-hospitalar, garantir otimização de recursos hospitalares mediante a crescente procura por atendimento no Departamento de Emergência (DE).

A descrição de experiências em gestão de serviços de saúde e a publicação desses dados propiciam a troca de conhecimentos entre os pesquisadores e fomentam dados para futuras análises.

\section{MÉTODO}

Este trabalho refere-se aos esforços de organização de demanda de atendimento durante a pandemia de COVID-19 iniciados no mês de março de 2020 e atualmente mantidos no DE do Hospital de Messejana Dr. Carlos Alberto Studart Gomes em Fortaleza, Ceará. Trata-se de um trabalho descritivo, a partir de um relato de experiência, realizado através da observação e discussão das estratégias de enfrentamento à pandemia de COVID-19 pela equipe de gestão do DE do referido hospital.

\section{RESULTADOS}

À medida que o paciente chega às dependências externas do DE, o primeiro atendimento é realizado em uma grande tenda ao ar livre, onde um enfermeiro realiza o acolhimento e a triagem verbal na investigação de sintomas gripais mais comuns (tosse, coriza, odinofagia, congestão nasal ou conjuntival, adinamia e produção de escarro) (Figura). Nesse ambiente foram demarcadas áreas no chão sinalizando os locais onde os pacientes deviam aguardar o atendimento inicial respeitando distanciamento de um metro dos outros pacientes. Estavam disponíveis também assentos respeitando as mesmas normatizações do distanciamento.
Antes da implementação desse fluxo, verificou-se o número médio de atendimentos por dia, levando-se em conta o dia da semana e horários específicos, e foi observado que um único profissional enfermeiro acolhedor, nessa função abreviada de indagar os pacientes sobres sintomas gripais, pudesse estabelecer uma boa vazão para a etapa de atendimento seguinte, de forma que um segundo profissional poderia ser acionado caso a demanda fosse acima do esperado. Desde o início da execução, observamos um andamento bastante favorável dentro do fluxo, ao ponto da não observância de aglomerações no local.

No momento de chegada dos pacientes, além de identificá-los com ou sem sintomas gripais, o enfermeiro acolhedor oferecia a eles máscara cirúrgica, álcool etílico em gel a $70 \%$ para as mãos, além de uma pulseira de identificação com cores diferentes para os dois tipos de perfis, suspeito e não suspeito de COVID-19, a depender dos sintomas gripais. A partir de então, os pacientes eram encaminhados para fluxos distintos, separados estruturalmente, com toda a equipe de profissionais assistenciais voltados especificamente para cada tipo de perfil. As medidas adotadas favoreciam um menor risco de contaminação entre os pacientes no geral, assim como garantiam uma maior segurança aos profissionais que usavam adequadamente os equipamentos de proteção individuais (EPI) a depender do perfil do paciente e do tipo de assistência oferecida.

Uma estrutura externa de atendimento para uma segunda triagem foi construída na frente da emergência, para o atendimento específico dos pacientes com sintomas gripais, objetivando a confirmação diagnóstica de síndrome gripal (febre de início súbito, mesmo que referida; tosse; odinofagia; e acompanhado de pelo menos um dos sintomas: cefaleia, mialgia ou artralgia, na ausência de outro diagnóstico) e da síndrome respiratória aguda grave (SRAG) por meio da identificação de dispneia, desconforto respiratório e oximetria de pulso com saturação de oxigênio menor que $95 \%{ }^{5}$. Nesse contexto, um médico e um enfermeiro se deslocavam através de quatro boxes de atendimento aos pacientes e com o auxílio de um sistema web acessado via tablets, as informações eram alimentadas e o direcionamento do fluxo e conduta já eram definidos de forma automática. Cabe ressaltar que por medidas de segurança todos os boxes continham dispensadores de álcool etílico em gel a 70\%, assim como estavam organizados mantendo um distanciamento de dois metros entre a cadeira do paciente e a mesa que o profissional se encontrava. 0 intuito desta etapa era definir a indicação de continuidade do atendimento intra-hospitalar, tendo em vista que boa parte desses pacientes tinham como indicação uma terapia domiciliar, evitando, portanto, um fluxo desnecessário dentro da emergência e contribuindo para um menor índice de transmissibilidade da doença.

Após passarem pela triagem dos sintomas gripais, os pacientes com critérios de avaliação mais detalhados e/ou maior gravidade eram encaminhados através de um fluxo único e distinto para o eixo interno COVID-19, onde eram avaliados em consultórios médicos ou na sala vermelha para definição de internação ou alta hospitalar. Aqueles que não tivessem essa indicação, na própria triagem já recebiam as devidas orientações quantos às terapias medicamentosas, atestado médico e aos cuidados gerais domiciliares. 


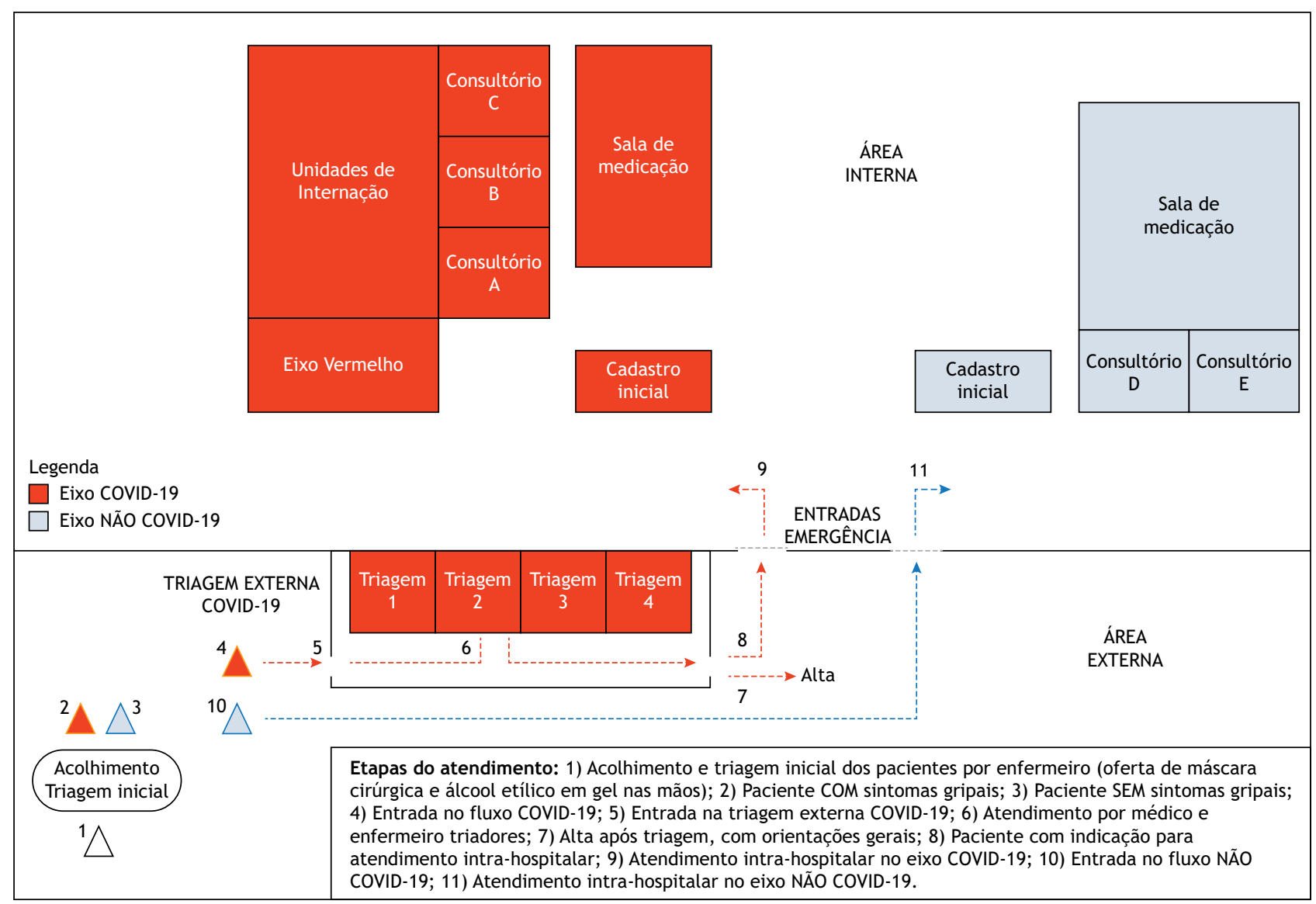

Fonte: Elaborada pelos autores, 2020.

Figura. Esquematização dos fluxos de atendimento na emergência de pacientes COVID-19 e não COVID-19.

Os pacientes que apresentassem sintomas não gripais, desde 0 atendimento inicial com o enfermeiro acolhedor, eram encaminhados para o eixo interno não COVID, que segue também um fluxo individualizado em uma área hospitalar estruturalmente distante do eixo interno COVID-19. Aqueles que chegavam à entrada da emergência sem qualquer tipo de sintoma, mesmo referindo contato prévio com paciente suspeito ou confirmado para COVID-19, era orientado pelo enfermeiro acolhedor da não indicação para o atendimento na emergência, recebendo um encarte com todas as informações gerais e cuidados domiciliares relacionados à doença. As definições dos fluxos e critérios das triagens foram embasadas nos protocolos e orientações propostas pelo MS, assim como na experiência dos gestores que compõe a emergência do hospital ${ }^{5}$.

Todos os profissionais assistentes do DE estavam paramentados com EPI de acordo com as definições institucionais da Comissão de Controle de Infecção Hospitalar (CCIH), amparadas pelas orientações da Agência Nacional de Vigilância Sanitária (Anvisa) através da Nota Técnica $n^{\circ}$ 04/20206. Para os atendimentos de triagem preliminar não envolvendo contato direto, os profissionais realizavam os questionamentos e observações com uma distância espacial de pelo menos um metro, portando máscara cirúrgica. Diante da necessidade do exame físico de pacientes com sintomas respiratórios, o profissional se paramenta com máscara cirúrgica, avental impermeável, luvas, gorro, proteção ocular e calçados fechados. Em todos os setores de atendimento ou circulação de pessoas, havia dispensadores com álcool etílico em gel a $70 \%$ e pias para lavagem das mãos em locais estratégicos.

As equipes de trabalho eram orientadas a realizarem turnos de $6 \mathrm{~h} /$ dia com carga horária máxima semanal de $30 \mathrm{~h}$, porém, devido à indisponibilidade de profissionais para atendimento às demandas gerais de pacientes COVID-19, tem-se observado a necessidade de aumento na carga horária semanal dos profissionais para garantir uma assistência adequada aos pacientes em todo o hospital.

\section{DISCUSSÃO}

Em se tratando de uma doença com alta taxa de transmissão a partir de aerossóis e fômites e tendo em superfícies e rotas de contato seus principais meios de transmissão, a pandemia de COVID-19 tem evoluído rapidamente pelo mundo ${ }^{7,8}$. No entanto, a transmissão de doenças respiratórias pode frequentemente ser reduzida através do controle de exposição por meio de gerenciamento, controles administrativos e uso de EPI'. Desta forma, cuidados com o fluxo de doentes suspeitos ou confirmados e o uso racional de EPI configuram pontos importantes da cadeia de 
disseminação da doença dentro e fora dos serviços de saúde. Torna-se papel do DE garantir que casos suspeitos sejam isolados para evitar contaminações dentro do ambiente hospitalar, protegendo trabalhadores da saúde e outros pacientes ${ }^{10}$.

Por se tratar de um hospital público terciário, especializado em doenças cardiopulmonares, com um histórico de alto fluxo de pacientes atendidos diariamente na emergência, bem como uma das referências no enfrentamento ao coronavírus a uma população de quase 2,7 milhões de habitantes, se fez necessária uma estratégia que garantisse um atendimento efetivo para os pacientes com e sem sintomas gripais, assim como segurança para toda equipe de profissionais.

Dado o risco de transmissão nosocomial, alguns serviços relataram a necessidade de separação de suas equipes em profissionais que atuam com pacientes infectados e profissionais que atuam em outras demandas hospitalares, como medida de redução de infecção?.

Durante uma pandemia, os trabalhadores da saúde submetem-se a longas jornadas de trabalho, demandas excessivas, recursos inadequados e riscos de contaminação pelo contato direto com pacientes infectados. A segurança dos trabalhadores da saúde e a motivação desses em continuar em seus postos de trabalho são um dos grandes impactos gerados pela pandemia de COVID-197. Durante a preparação das equipes de saúde, simulações in situ apresentam-se como estratégia de reconhecer falhas nos fluxos operacionais e entender os desafios de trabalhar em ambientes e com EPI não familiares a rotina das equipes ${ }^{9}$.

$O$ atendimento do paciente em um DE necessita ser diferenciado, objetivando sua criticidade, porém sem perder sua integralidade e segurança. Os otimistas em tecnologia da saúde sugerem que o DE é um local singular para se beneficiar de um sistema eletrônico para gerenciamento dos pacientes ${ }^{11}$. As ferramentas tecnológicas dentro do ambiente hospitalar estão se tornando cada vez mais importantes, em vista da demanda por um serviço mais organizado e eficiente. 0 uso de plataformas informatizadas ajuda a alinhar as informações dentro da equipe, contenção de gastos e melhorias na qualidade de assistência ao paciente ${ }^{12}$.

Através de um sistema de gestão efetivo é possível tomar decisões assertivas e gerenciar melhor o paciente dentro do ambiente hospitalar ${ }^{13}$. Além de diminuir os custos com recursos humanos, papel e local de armazenamento. Com as informações geradas por sistema informatizado, a análise de dados e indicadores dentro do hospital facilita a identificação do desempenho e falhas nos processos ${ }^{14}$. Além disso, as trocas de informações em saúde e análises em nível de população ou comunidade impulsionam a colaboração às melhorias do sistema de saúde como um todo ${ }^{15}$.

A triagem extra-hospitalar e adequação dos fluxos hospitalares com a diferenciação de um eixo não COVID e um eixo COVID representa o alicerce das medidas de adequação da coordenação da emergência. A observação e a ativa participação na implementação dessa estratégia permitiram o registro desta experiência que, até o momento da produção deste trabalho, mantém-se em funcionamento.

\section{CONCLUSÕES}

O início da atenção aos pacientes nos serviços de saúde deve garantir fluxos claros para separar aqueles com sintomas gripais sugestivos de COVID-19 dos demais usuários do serviço. Essa abordagem inicial perpassa pela garantia de segurança aos usuários e aos trabalhadores da saúde, e proporciona adequado dimensionamento ao uso de recursos humanos e materiais na emergência.

A criação de uma triagem extra-hospitalar utilizando uma ferramenta informatizada pode ser evidenciada como uma estratégia na otimização dos recursos hospitalares, evitando a admissão de pacientes que poderiam ter suas demandas atendidas em serviços de menor complexidade ou mesmo em seus domicílios. Essa ferramenta proporcionou maior agilidade na coleta de dados dos pacientes que procuraram o serviço, orientou os fluxos de atendimento do hospital e permitiu armazenamento dos dados para produção de estudos posteriores.

\section{REFERÊNCIAS}

1. Ezequiel J, Persad G, Upshur R, Thome B, Parker M, Glickman A et al. Fair allocation of scarce medical resources in the time of COVID-19. N Engl J Med. 2020;382(21):2049-55. https://doi.org/1056/NEJMsb2005114

2. Werneck GL, Carvalho MS. A pandemia de COVID-19 no Brasil: crônica de uma crise sanitária anunciada. Cad Saude Publica. 2020;36(5):1-4. https://doi.org/10.1590/0102-311×00068820

3. Ministério da Saúde (BR). COVID-19 painel coronavírus. Coronavírus Brasil. 16 maio 2020[acesso 16 maio 2020]. Disponível em: https://covid.saude.gov.br/

4. Agência Nacional de Saúde Suplementar - ANS. Beneficiários de planos privados de saúde, por cobertura assistencial (Brasil 2010-2020). Rio de Janeiro: Agência Nacional de Saúde Suplementar; 2020[acesso 10 maio 2020]. Disponível em: https://www.ans.gov.br/perfil-do-setor/dados-gerais
5. Secretaria de Atenção Primária à Saúde - SAPS. Estratificação da gravidade da síndrome gripal. In: Secretaria de Atenção Primária à Saúde - SAPS. Protocolo de manejo clínico do coronavírus (COVID-19) na atenção primária à saúde. Brasília; Ministério da Saúde; 2020[acesso 27 maio 2020]. p. 9-18. Disponível em: saude.gov.br/images/pdf/2020/ marco/20/20200318-ProtocoloManejo-ver002.pdf

6. Agência Nacional de Vigilância Sanitária - ANVISA. Orientações para serviços de saúde: medidas de prevenção e controle que devem ser adotadas durante a assistência aos casos suspeitos ou confirmados de infecção pelo novo Coronavírus (SARS-CoV-2). Brasília: Agência de Vigilância Sanitária; 2020 [acesso 27 maio 2020]. 92 p. Disponível em: http://portal.anvisa.gov.br/documents/33852/271858/ Nota+Técnica+n+04-2020+GVIMS-GGTES-ANVISA/ ab598660-3de4-4f14-8e6f-b9341c196b28 
7. Schwartz J, King C, Yen M. Protecting healthcare workers during the coronavirus disease 2019 (COVID-19) outbreak: lessons from Taiwan's severe acute respiratory syndrome response. Clin Infect Dis. 2020:1-3. https://doi.org/10.1093/cid/ciaa255

8. Cook TM. Personal protective equipment during the coronavirus disease (COVID) 2019 pandemic: a narrative review. Anaesthesia. 2020:1-8. https://doi.org/10.1111/anae.15071

9. Wong J, Goh QY, Tan Z, Lie SA, Tay YC, Ng SY et al. Preparing for a COVID-19 pandemic: a review of operating room outbreak response measures in a large tertiary hospital in Singapore. Can J Anaesth. 2020;67(6):732-45. https://doi.org/10.1007/s12630-020-01620-9

10. Wee LE, Fua TP, Chua YY, Ho AFW, Sim XYJ, Conceicao EP et al. Containing COVID-19 in the emergency department: the role of improved case detection and segregation of suspect cases. Acad Emerg Med. 2020;27(5):379-87. https://doi.org/10.1111/acem.13984
11. Selck FW, Decker SL. Health information technology adoption in the emergency department. Health Serv Res. 2016;51(1):32-47. https://doi.org/10.1111/1475-6773.12307

12. Escher A, Hainc N, Boll D. Business intelligence in hospital management. Radiol Manage. 2016;38(3):47-52.

13. Farias DC, Araujo FO. Gestão hospitalar no Brasil: revisão da literatura visando ao aprimoramento das práticas administrativas em hospitais. Cienc Saude Coletiva. 2017;22(6):1895-904. https://doi.org/10.1590/1413-81232017226.26432016

14. David Y, Jahnke EG. Planning hospital medical technology management. IEEE Eng Med Biol Mag. 2004;23(3):73-9. https://doi.org/10.1109/MEMB.2004.1317985

15. Gamache R, Kharrazi H, Weiner JP. Public and population health informatics: the bridging of big data to benefit communities. Yearb Med Inform. 2018:27(1):199-206. https://doi.org/10.1055/s-0038-1667081

Contribuição dos Autores

Oliveira BDD - Concepção, planejamento (desenho do estudo), aquisição, análise, interpretação dos dados e redação do trabalho. Khoury SH, Martins VG - Aquisição, análise, interpretação dos dados e redação do trabalho. Arnaud FCS, Rabêlo DRV - Aquisição, análise e interpretação dos dados. Gaspardi AC - Redação do trabalho. Todos os autores aprovaram a versão final do trabalho.

Conflito de Interesse

Os autores informam não haver qualquer potencial conflito de interesse com pares e instituições, políticos ou financeiros deste estudo.

Esta publicação está sob a licença Creative Commons Atribuição 3.0 não Adaptada.

Para ver uma cópia desta licença, visite http://creativecommons.org/licenses/by/3.0/deed.pt_BR. 